\title{
The Escherichia coli heat-labile enterotoxin B subunit protects from allergic airway disease development by inducing $\mathrm{CD}^{+}$ regulatory $T$ cells
}

\author{
DS Donaldson ${ }^{1}, M_{\text {Apostolaki }}{ }^{1}$, HK Bone ${ }^{1}$, CM Richards ${ }^{1}$ and NA Williams ${ }^{1}$
}

The B subunit of $E$. coli heat-labile enterotoxin (EtxB) protects against the development of Thelper type 1 (Th1)-mediated autoimmune pathologies in mice. Protection was transferable with splenic CD4 ${ }^{+}$Tcells and was less effective following CD25 depletion; implying a Tregulatory cell (Treg)-mediated process. We hypothesized that if this were the case, then EtxB would also control a Th2-mediated disorder. We tested the effect of EtxB treatment on asthma development in ovalbumin (OVA)-sensitized mice. EtxB treatment diminished eosinophilia in bronchoalveolar lavage samples, reduced OVA-specific immunoglobulin $E$ and interleukin 4 production locally and systemically, and reduced airway hyperreactivity. EtxB induced a dose-dependent increase in Foxp3 ${ }^{+} \mathrm{CD} 4^{+} \mathrm{T}$ cells, and adoptive transfer of splenic $\mathrm{CD}^{+}{ }^{+}$T cells partially suppressed lung pathology. Importantly, EtxB treatment increased OVA-specific CD4 ${ }^{+}$Foxp3 $^{+}$ Tcells in the lung and systemically. These data demonstrate that EtxB modulates the differentiation of allergen-specific T cells causing inducible Treg induction and preventing disease.

\section{INTRODUCTION}

Asthma is a chronic inflammatory disease of the airways characterized by airway hyper-responsiveness, the prevalence of which has increased markedly in the past 20 years. ${ }^{1}$ The pathogenesis of asthma is highly complex, involving multiple innate and adaptive immune components. Classically, disease results from allergic responses to innocuous antigens resulting in polarized $\mathrm{T}$ helper type 2 (Th2) responses and allergen-specific immnuglobulin E (IgE) production. ${ }^{2}$ Current treatment protocols revolve around symptomatic relief in mild and moderate asthma. Newer therapies targeting $\operatorname{IgE}$ and leukotriene receptors are only used in limited patient groups. Although current approaches may effectively deal with the symptoms of disease, ${ }^{3}$ none address the underlying immunological processes driving pathology. The use of allergen in approaches that seek to rebalance the immune response carries significant risk and the efficacy of "desensitization" therapies is controversial. Approaches that induce tolerance to causative allergens without exposing patients to native allergen would therefore be an ideal method of addressing the basic processes driving the immune response in allergic asthma and have the potential to act as a truly disease-modifying therapy.

Since the discovery of subsets of $\mathrm{CD} 4{ }^{+} \mathrm{T}$ cells that are capable of suppressing antigen-specific immune responses, termed regulatory $\mathrm{T}$ cells (Treg), ${ }^{4}$ these cells have become the focus of much research into novel immunotherapies for asthma. ${ }^{5}$ Allergen peptide derivatives, which by-pass safety issues associated with the administration of whole allergens, have shown some efficacy in treating asthma-related symptoms in mice ${ }^{6,7}$ and humans ${ }^{8}$ through a mechanism that may involve modulation of Treg. ${ }^{9}$ Interestingly, peptide-treated patients also display diminished responses to additional epitopes of allergens from which the peptide was derived, ${ }^{10}$ suggesting the induction of bystander suppression. Importantly, peptides from only a limited number of defined allergens have been tested so far $^{11}$ and while they may be effective in patients with known allergen-sensitivity and HLA (human leukocyte

${ }^{1}$ Cellular and Molecular Medicine, School of Medical Sciences, University of Bristol, Bristol, UK. Correspondence: NA Williams, (neil.a.williams@bristol.ac.uk) Received 23 December 2011; accepted 18 August 2012; published online 3 October 2012. doi:10.1038/mi.2012.93 
antigen) background, these therapies are potentially confounded by the complex etiology of human asthmatic disease. ${ }^{12}$

Mucosal administration of the B subunit of the Escherichia coli heat-labile enterotoxin (EtxB) prevents autoimmune disease development by inducing Treg without the need for antigen co-administration. ${ }^{13,14}$ The E. coli heat-labile enterotoxin is a hetero-oligomeric complex composed of an enzymatic A subunit and five identical B subunits and is a close relative of cholera toxin. ${ }^{15}$ The B subunit mediates cellular entry via binding to $G_{M 1}$ ganglioside present in the cell membrane, whereas the A subunit is responsible for toxicity. ${ }^{16,17}$ Recombinant EtxB lacks toxin activity but has a potent immuno-modulatory capacity. In NOD mice, a murine model of type 1 diabetes (T1D), intranasal EtxB alone prevented diabetes development. ${ }^{14}$ Similarly, intragastric or intranasal EtxB treatment was sufficient to prevent disease development in a murine model of rheumatoid arthritis, collagen-induced arthritis (CIA). ${ }^{13}$ Protection from both the CIA and T1D development was transferable with $\mathrm{CD}^{+}{ }^{+} \mathrm{T}$ cells from the spleen, suggesting that EtxB modulated a population of Treg. Further analysis revealed that EtxB induces an antigen-specific increase in Foxp3-expressing $\mathrm{CD}^{+}{ }^{+}$Treg. ${ }^{18}$

The process by which EtxB modulates Treg is unclear. Our recent studies have sought to determine the effects of EtxB in vivo to determine its mechanism of action. ${ }^{18}$ We have shown that EtxB treatment induces an increase in interleukin (IL)-10 and transforming growth factor (TGF)- $\beta_{1}$ production by epithelial cells at the site of delivery and by macrophages in lymphoid tissues. These cytokines are associated with modulating $\mathrm{T}$-cell differentiation in order to give rise to antigenspecific $\mathrm{CD}^{+}{ }^{+}$Foxp $^{+}$Treg and are not consistent with promotion of a Th2 response, as had been suggested previously when upregulation of collagen-specific $\operatorname{IgG}_{1}$ levels was observed following high dose treatment with EtxB intranasally. ${ }^{13}$ However, it remains unclear whether EtxB could be effective in modulating a Th2-driven pathology such as asthma.

If the underlying mechanism of EtxB action is the induction of increased Treg, EtxB may also have therapeutic benefit in Th2-mediated disorders, such as asthma. Testing this has the potential both to clarify the relative involvement of Treg vs. Th2 cells in the mechanism of action, and also pointing toward a novel therapy for an important human disease. Therefore, the effect of intranasal EtxB treatment in the widely used ovalbumin (OVA) asthma model was determined. EtxB treatment suppressed asthma development, controlling all of the hallmark features of the disease. The mechanism by which EtxB altered asthma was also investigated by studying its effects on Foxp3 expression within polyclonal and allergenspecific $\mathrm{CD}^{+}{ }^{+} \mathrm{T}$-cell populations.

\section{RESULTS}

Intranasal treatment with EtxB reduces eosinophilia and enhanced pause (Penh) in OVA-sensitized mice

To determine whether EtxB was capable of suppressing a Th2mediated disease, the effect of intranasal treatment with EtxB on asthma development in OVA-sensitized mice was assessed.
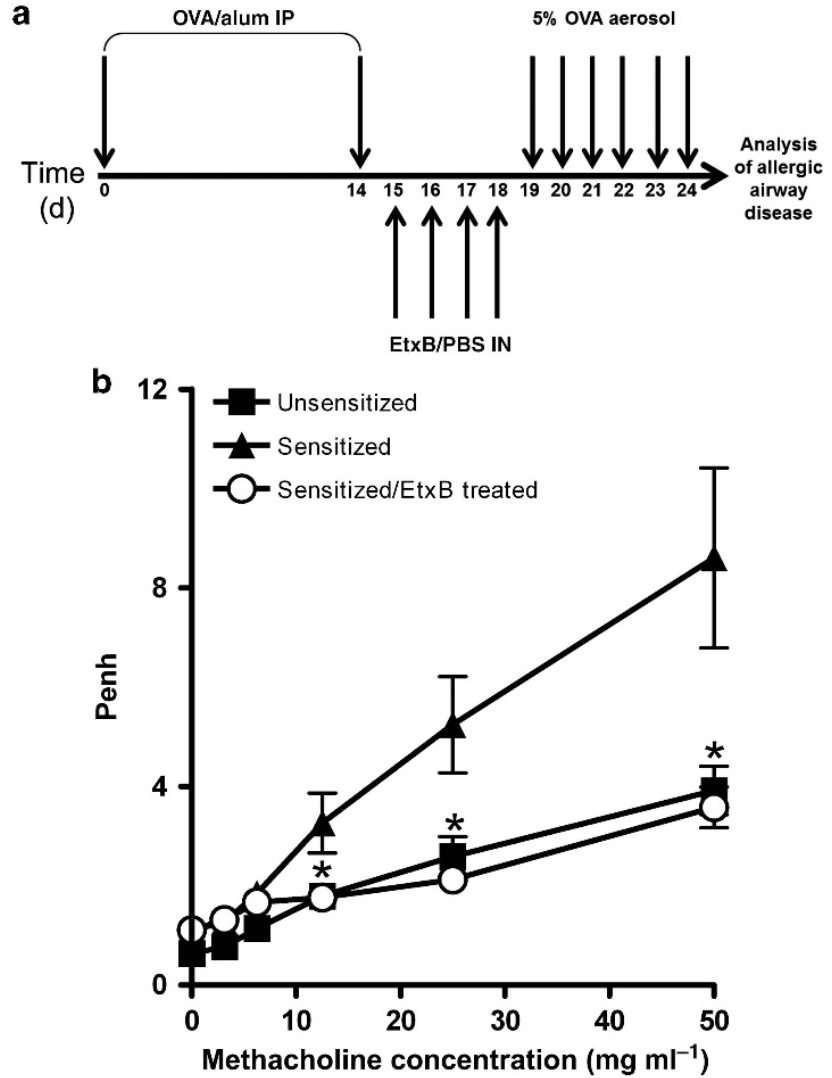

Figure 1 The effect of intranasal (IN) treatment with EtxB (B subunit of E. coli heat-labile enterotoxin) on airway hyper-responsiveness (AHR). (a) Mice were sensitized with ovalbumin (OVA)/alum followed by treatment with $20 \mu \mathrm{g} \mathrm{EtxB}$ on 4 consecutive days. Mice were then challenged with $5 \%$ OVA aerosol for 6 consecutive days. Untreated sensitized and unsensitized mice were included as controls. (b) Following challenge, enhanced pause (Penh) values, a measure of AHR, were determined by recording respiratory pressure curves by unrestrained whole-body plethysmography in response to increasing concentrations of methacholine. The data shown are representative of five similar experiments, values represent mean \pm s.e.m. $(n=6)$. Statistical differences were determined using Student's $t$-test, ${ }^{*} P<0.05$. $\mathrm{IP}$, intraperitoneal; PBS, phosphate-buffered saline.

Mice were sensitized with OVA and then either left untreated or given $20 \mu \mathrm{g}$ of EtxB intranasally for 4 consecutive days, before being challenged with aerosolized OVA daily for 6 days (Figure 1a). Unsensitized mice were also challenged with OVA. Penh was determined in response to increasing concentrations of methacholine using unrestrained whole-body plethysmography. OVA-sensitized and challenged mice showed clear evidence of increased methacholine sensitivity in comparison to unsensitized OVA-challenged animals, evidenced by the more rapid and greater increase in Penh following exposure (Figure 1b). Intranasal treatment with EtxB significantly reduced the Penh of OVA-sensitized and challenged mice to such an extent that it became indistinguishable from the negative control. The lipopolysaccharide content of EtxB was $<0.6 \mathrm{EU} /$ dose and administration of EtxB alone did not induce inflammatory airway disease (see Supplementary Figure S1 online). 

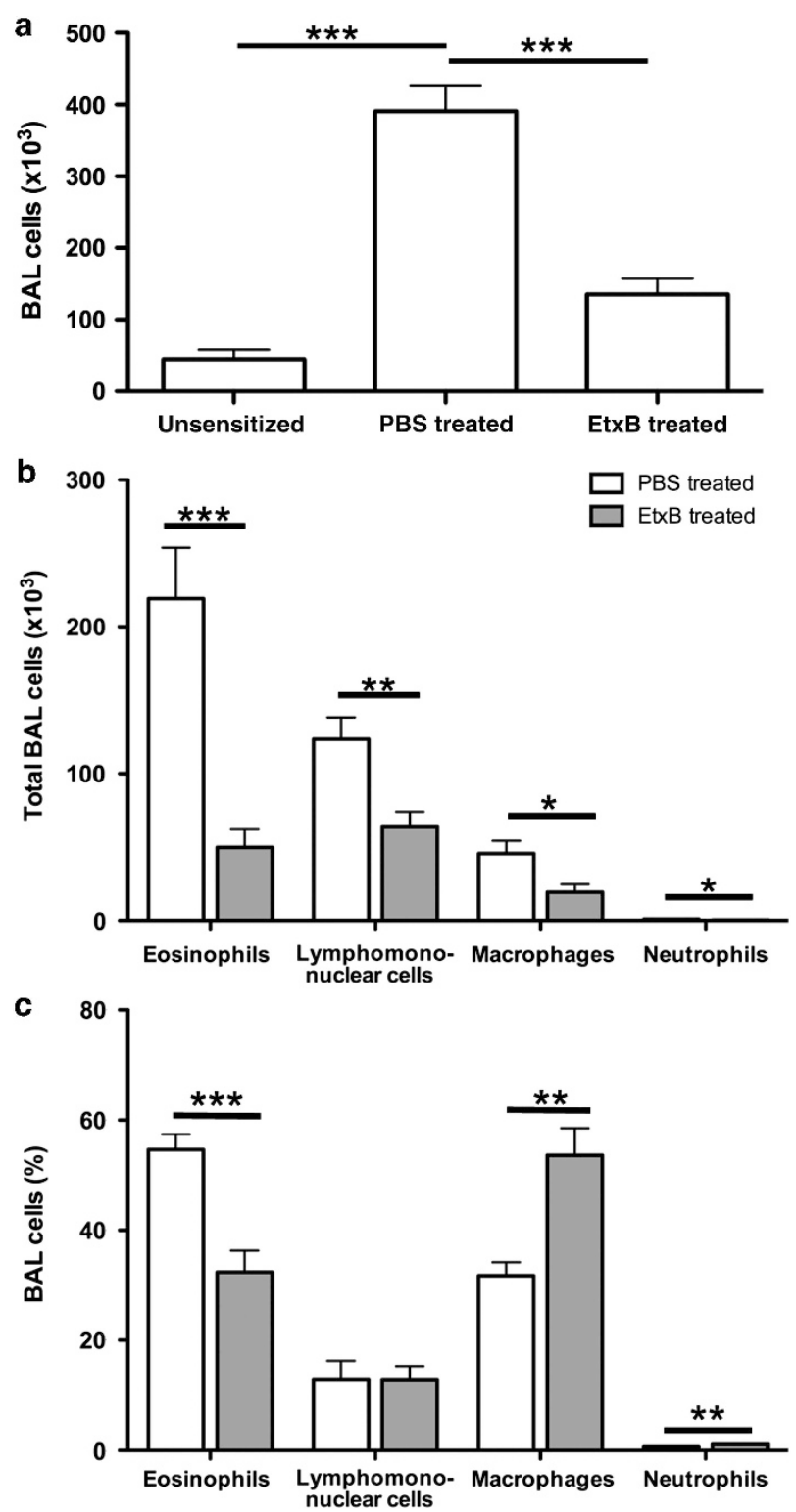

Figure 2 The effect of intranasal treatment with EtxB (B subunit of $E$. coli heat-labile enterotoxin) on bronchoalveolar lavage (BAL) cells. Mice were sensitized with ovalbumin (OVA)/alum followed by treatment with $20 \mu \mathrm{g}$ EtxB or an equivalent volume of phosphate-buffered saline (PBS) on 4 consecutive days. Mice were then challenged with $5 \%$ OVA aerosol for 6 consecutive days. Following challenge, (a) BAL cells were removed and counted. Unsensitized mice were included as controls. Cytospins were prepared, stained with Leishmans stain and (b) the total cell number and (c) percentage of eosinophils, macrophages, neutrophils and lymphomononuclear cells were determined by microscopy. The data shown are representative of two similar experiments, bars represent mean \pm s.e.m. $(n=8)$. Statistical differences were determined using Student's $t$-test, ${ }^{\star \star *} P<0.001,{ }^{* \star} P<0.01$, ${ }^{*} P<0.05$.

Bronchoalveolar lavage (BAL) samples were collected from the animals after Penh measurement and cell numbers were determined. The significant reduction in Penh was associated with a significant reduction in the total number of cells in BAL samples (Figure 2a). This reduced cell count after treatment was associated with a significant reduction in the dominant eosinophil infiltrate as well as lymphomononuclear cells, macrophages, and neutrophils (Figure $\mathbf{2 b}, \mathbf{c}$ ). Therefore, intranasal treatment with EtxB effectively suppresses the development of asthma in OVA-sensitized mice, shown by the reduction in airway hyper-responsiveness and inflammatory infiltration of the airways.

\section{Protection from asthma is associated with reduced local and systemic IL-4 and IgE production}

Sensitization with OVA/alum results in the generation of polarized Th2 responses characterized by increased IL-4 production, important for the development of eosinophilia and OVA-specific IgE. ${ }^{19}$ The effects of EtxB on IL-4, as well as the immuno-regulatory cytokine IL-10 and the Th1-associated cytokine interferon $\gamma$ (IFN $\gamma$ ) was measured in BAL samples. EtxB treatment significantly reduced the IL- 4 concentration in the BAL in comparison to that found in samples from phosphate-buffered saline (PBS)-treated animals (Figure 3a). The reduction in IL-4 levels was not matched by lower quantities of the other cytokines measured. IFN $\gamma$ levels were similar in all the three groups tested, and although mean levels of IL-10 were higher in the EtxB-treated group the difference was not significant. Splenocytes were isolated from EtxB and PBS-control-treated mice and cultured with or without OVA in order to measure the levels of antigen-driven proliferation and cytokine production. OVA-specific proliferation (Figure $\mathbf{3 b}$ ) and cytokine production (Figure 3c) by splenocytes from EtxB-treated mice were significantly reduced.

IL-4 is a key cytokine in promoting isotype switching to IgE, a key antibody class in allergy, although not critical to the development of disease in the murine OVA-asthma model. ${ }^{20}$ Anti-OVA IgE levels were also assessed in the BAL and serum samples of treated and untreated mice by ELISA (enzymelinked immunosorbent assay). In addition, the Th1- and Th2-associated IgG subclasses, $\operatorname{IgG}_{2 \mathrm{a}}$ and $\mathrm{IgG}_{1}$, respectively, were also assessed in the serum. In the BAL (Figure 4a), antiOVA IgE levels were significantly reduced by EtxB treatment. A similar trend was also observed in the serum (Figure $\mathbf{4 b}$ ) associated with decreased $\operatorname{IgG}_{2 \mathrm{a}}$ levels (Figure 4c). By contrast, anti-OVA $\operatorname{IgG}_{1}$ levels in the serum were not affected by EtxB treatment (Figure 4c).

\section{EtxB treatment reduces OVA-specific $\mathrm{CD} 4^{+}$T-cell infiltration into the lung}

The effect of EtxB treatment on allergen-specific $\mathrm{CD} 4{ }^{+} \mathrm{T}$-cell activation and differentiation was assessed by adoptively transferring $\mathrm{CD}^{+} \mathrm{T}$ cells expressing an OVA-specific transgenic TCR (T-cell receptor) into naive BALB/c mice on the day of the second OVA sensitization. Recipients were treated as before with intranasal EtxB or PBS for 4 consecutive days, followed by 6 consecutive days of aerosol OVA challenge. After the final challenge, mice were culled and lung mononuclear cells isolated and analyzed by flow cytometry. In agreement with the previous analysis of BAL cell numbers, there was clear evidence that EtxB treatment reduced numbers of inflammatory cells in the lung tissue (data not shown). The overall decrease in cell infiltration was matched by a significant 
a

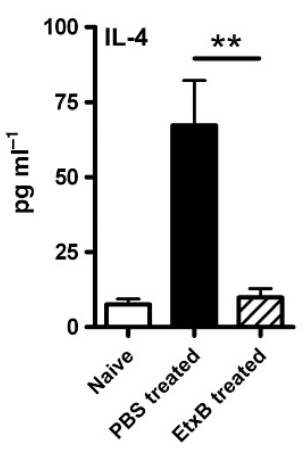

C

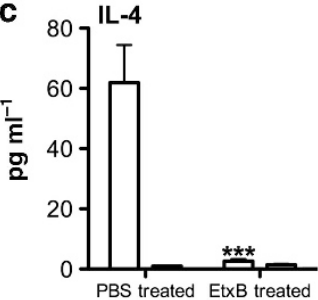

Waive PBS treated
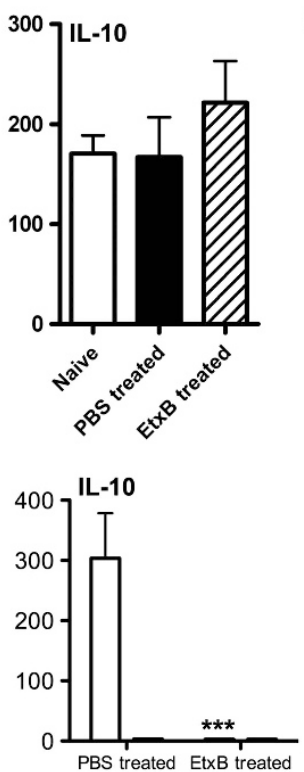

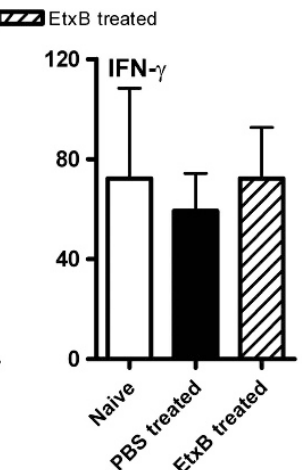

b

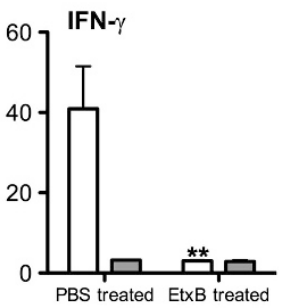

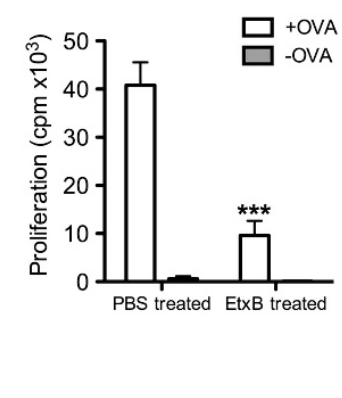

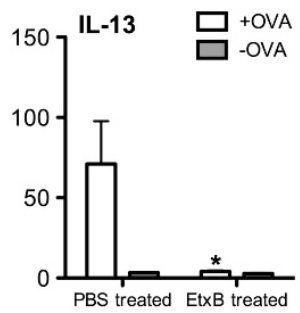

Figure 3 The effect of intranasal treatment with EtxB (B subunit of $E$. coli heat-labile enterotoxin) on cytokine production. Mice were sensitized with ovalbumin (OVA)/alum followed by treatment with $20 \mu \mathrm{g} \mathrm{EtxB}$ or an equivalent volume of phosphate-buffered saline on 4 consecutive days. Mice were then challenged with $5 \%$ OVA aerosol for 6 consecutive days. Following challenge, BAL cells were removed and the supernatants isolated. (a) The concentration of interleukin (IL)-4, IL-10, and interferon (IFN)- $\gamma$ was determined by enzyme-linked immunosorbent assay. Bars represent mean \pm s.e.m. $(n=10)$. Unsensitized mice were included as controls. Spleen cells were also isolated and re-stimulated in vitro in the presence or absence of OVA. Following 3 days culture, (b) proliferation was assessed and (c) supernatants were isolated and the concentration of IL-4, IL-10 and IFN- $\gamma$ was determined by Luminex $(n=3)$. The data shown are representative of two similar experiments. Statistical differences were determined using Student's $t$-test; ${ }^{\star} P<0.05$, ${ }^{\star *} P<0.01$, and ${ }^{\star * \star} P<0.001$.

decrease in the number of OVA-specific $\mathrm{CD} 4^{+} \mathrm{KJ} 1-26^{+}$cells in the lungs (Figure 5a). Analysis of cytokine expression in OVA-specific T cells showed that the reduction in overall cell numbers was associated with a reduction in the numbers of cells expressing a range of key inflammatory cytokines. As shown in Figure $\mathbf{5 b}$, although similar proportions of cytokine-secreting cells were observed in the lung, lower absolute numbers of cells producing IL-4, IL-5, IL-13 as well as IFN- $\gamma$, IL-17, and IL-10 were found in the lungs of treated animals, correlating with the reduced IL-4 levels in the BAL; however, not with IFN- $\gamma$ or IL10 levels, suggesting that they are predominantly non-T cell derived. Similar results were obtained with cells from the draining mediastinal lymph nodes (dMLN).

Repeat experiments were carried out in which transferred OVA-specific CD4 ${ }^{+}$T cells were labeled with CFSE (carboxyfluorescein succinimidyl ester) in order to determine whether the lower number of OVA-specific T cells in the lungs of EtxBtreated mice is a result of reduced proliferation of infiltrating cells, or a reduction in infiltration of cells that had divided elsewhere. The majority of the OVA-specific cells entering the lung in untreated and EtxB-treated animals were highly divided, compared with those found in the dMLN (Figure 5c). EtxB treatment had only a modest effect on the proliferation of OVA-specific $\mathrm{CD} 4^{+} \mathrm{T}$ cells in the lung and dMLN, suggesting that reduced infiltration was the cause of the reduced number of cells present in the lung following EtxB treatment.
The modulation of autoimmune disease by EtxB is not dependent on local delivery of the molecule, but has instead been linked to the generation of Treg capable of entering sites of inflammation. Althoguh this may also be the mechanism underlying the effects observed on asthma, it is also possible that EtxB is entering the lung after intranasal administration and conditioning against the asthmatic response. Therefore, the effect of EtxB treatment on the number of cells in the lung pre-aerosol challenge was investigated. EtxB treatment induced a significant increase in lung cellularity (Figure 5d). This increase in the absence of aerosol challenge suggests that EtxB directly modulates the lung environment, potentially creating conditions that prevent the infiltration that is associated with pathology.

\section{Adoptive transfer of $\mathrm{CD}^{+}{ }^{+} \mathrm{T}$ cells from OVA-sensitized and EtxB-treated mice inhibits lung inflammation and hyper-responsiveness}

The contribution of direct effects of EtxB on the lung in mediating protection from asthma development was assessed using adoptive transfer. In both CIA and T1D, the site of pathology was distinct from the site of EtxB administration and protection was transferable with $\mathrm{CD} 4{ }^{+} \mathrm{T}$ cells. ${ }^{13,14}$ A similar adoptive transfer approach was used in order to determine whether the direct effect of EtxB treatment on the lung was critical in mediating protection from asthma in the mouse model. Briefly, mice were sensitized to OVA by two injections 

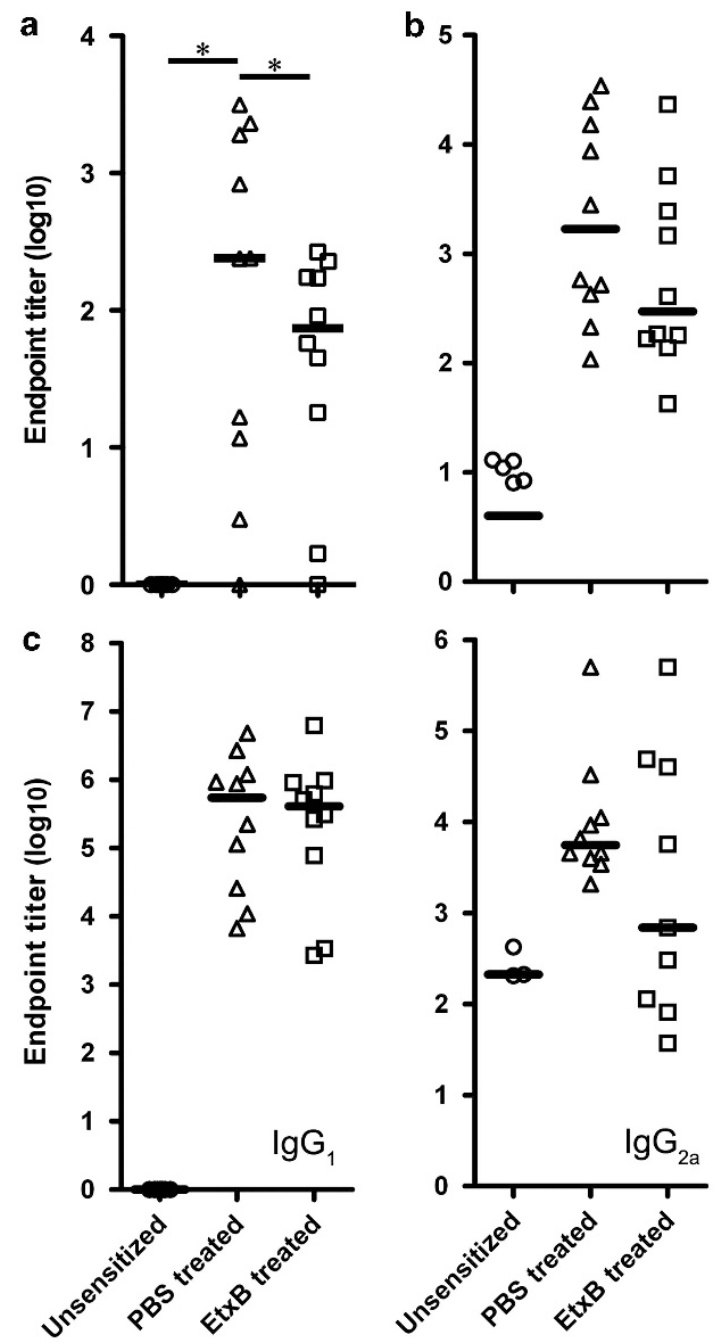

Figure 4 The effect of intranasal treatment with EtxB (B subunit of $E$. coli heat-labile enterotoxin) on antibody production. Mice were sensitized with ovalbumin (OVA)/alum followed by treatment with $20 \mu \mathrm{g}$ EtxB or an equivalent volume of phosphate-buffered saline (PBS) on 4 consecutive days. Mice were then challenged with 5\% OVA aerosol for 6 consecutive days. Following challenge, bronchoalveolar lavage cells were removed and the supernatants isolated. (a) The endpoint titers of OVA-specific immunoglobulin $E(\operatorname{lgE})$ were determined by enzyme-linked immunosorbent assay. (b) OVA-specific $\operatorname{lgE}$, (c) $\operatorname{lgG}_{1}$, and $\lg _{2 a}$ endpoint titers were also measured in serum. Lines represent median $(n=10)$, statistical differences were determined using Student's $t$-test, ${ }^{*} P<0.05$. Similar results were obtained in a separate study.

of OVA/alum followed by intranasal treatment with either EtxB or PBS for 4 consecutive days. After 24 days, splenic CD4 ${ }^{+} \mathrm{T}$ cells were purified and adoptively transferred into recipient OVA-sensitized mice on the day of the second intraperitoneal sensitization. Recipient mice were then challenged with aerosolized OVA for 6 consecutive days (Figure 6a). Unsensitized and sensitized mice that did not receive adoptively transferred cells were included as controls. Following challenge, Penh and BAL composition were assessed as previously described. The adoptive transfer of cells from PBS-treated mice resulted in a slight increase in Penh when compared with the no transfer group, presumably due to the presence of increased numbers of pathogenic OVA-specific $\mathrm{CD} 4^{+} \mathrm{T}$ cells (Figure $6 \mathbf{b}$ ). By contrast, transfer of cells from EtxB-treated mice partially protected mice from the disease, as revealed by a lowered Penh response than that of the PBS-transfer and the no transfer group.

The total number of cells present in the BAL correlated with the Penh value for each group (Figure 6c). An increased number of cells were seen in mice that had received cells from PBS-treated mice compared with the no transfer group, whereas a lower number of cells were observed in the mice that had received cells from EtxB-treated mice. The number of eosinophils, macrophages/neutrophils, and lymphomononuclear cells were determined in each of the BAL samples. Similar numbers of macrophages and neutrophils were observed in all the samples (Figure 6d). A similar number of lymphomononuclear cells were observed in both no transfer and EtxB-treated transfer, whereas transfer of cells from PBStreated mice induced a higher number of lymphomononuclear cells in the BAL. Interestingly, athough PBS-treated transfer increased the number of eosinophils, EtxB-treated transfer induced a reduction of the number of eosinophils. Taken together, these results suggest that the adoptive transfer of cells from EtxB-treated mice reduces airway hyper-responsiveness and eosinophilia and is therefore capable of partially preventing asthma development.

\section{Intranasal EtxB induces an increase in CD4 ${ }^{+}$Foxp3 $^{+}$ $T$ cells that is both antigen and dose-dependent in a Th2 stimulating context}

The ability of CD4 ${ }^{+} \mathrm{T}$ cells to transfer protection is suggestive of Treg induction, previously demonstrated by increased Foxp $3^{+}$Treg in the context of autoimmune disease modulation in DBA/ 1 mice by EtxB. ${ }^{18}$ Flow cytometric analysis showed EtxB treatment clearly induced a small but significant increase in the proportion of Foxp3-expressing $\mathrm{CD} 4{ }^{+} \mathrm{T}$ cells in both the cervical lymph nodes (CLN) and the spleen of BALB/c mice (Figure 7a,b), comparable with previous observations and confirming this effect was not strain specific. In order to determine whether this increase was dose-dependent, EtxB was given intranasally at varying concentrations to naive and OVA-sensitized mice on 4 consecutive days. On day 5 following treatment, spleens were removed and Foxp3 expression analyzed by flow cytometry. Intranasal EtxB treatment clearly induced a dose-dependent increase in the proportion of Foxp3-expressing $\mathrm{CD}^{+}{ }^{+} \mathrm{T}$ cells in the spleen of both the naive and OVA-sensitized mice (Figure 7b). Interestingly, this increase was associated with a significant increase in $\mathrm{CD} 4{ }^{+} \mathrm{CD} 25^{+}$Foxp $^{+}$T cells (Figure 7c). Similar results were also observed in the peripheral blood, demonstrating that these cells were not restricted to lymphoid tissues and could migrate to inflammatory sites.

Our results clearly demonstrate that EtxB induced an increase in the proportion of $\mathrm{CD}^{+} \mathrm{T}$ cells that express Foxp3 both in the absence or presence of OVA. We therefore tested whether this population included cells that are OVAspecific, and whether modulation of the "pathogenic" T-cell 

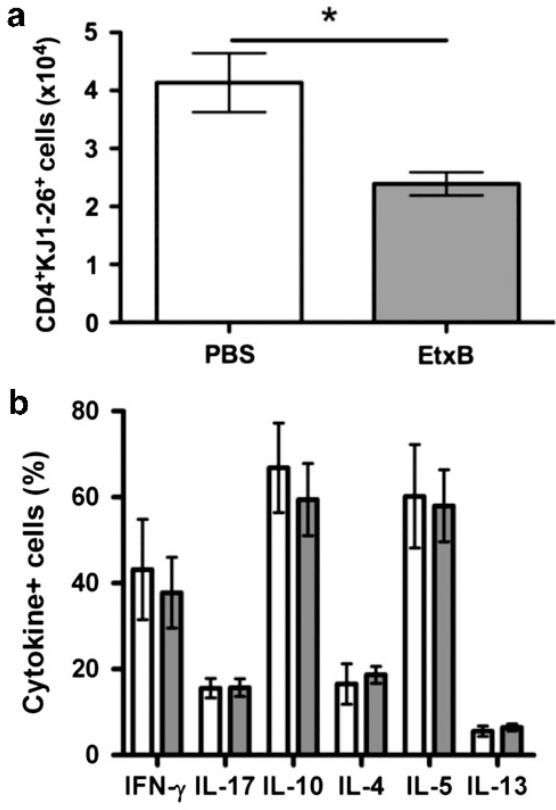

C
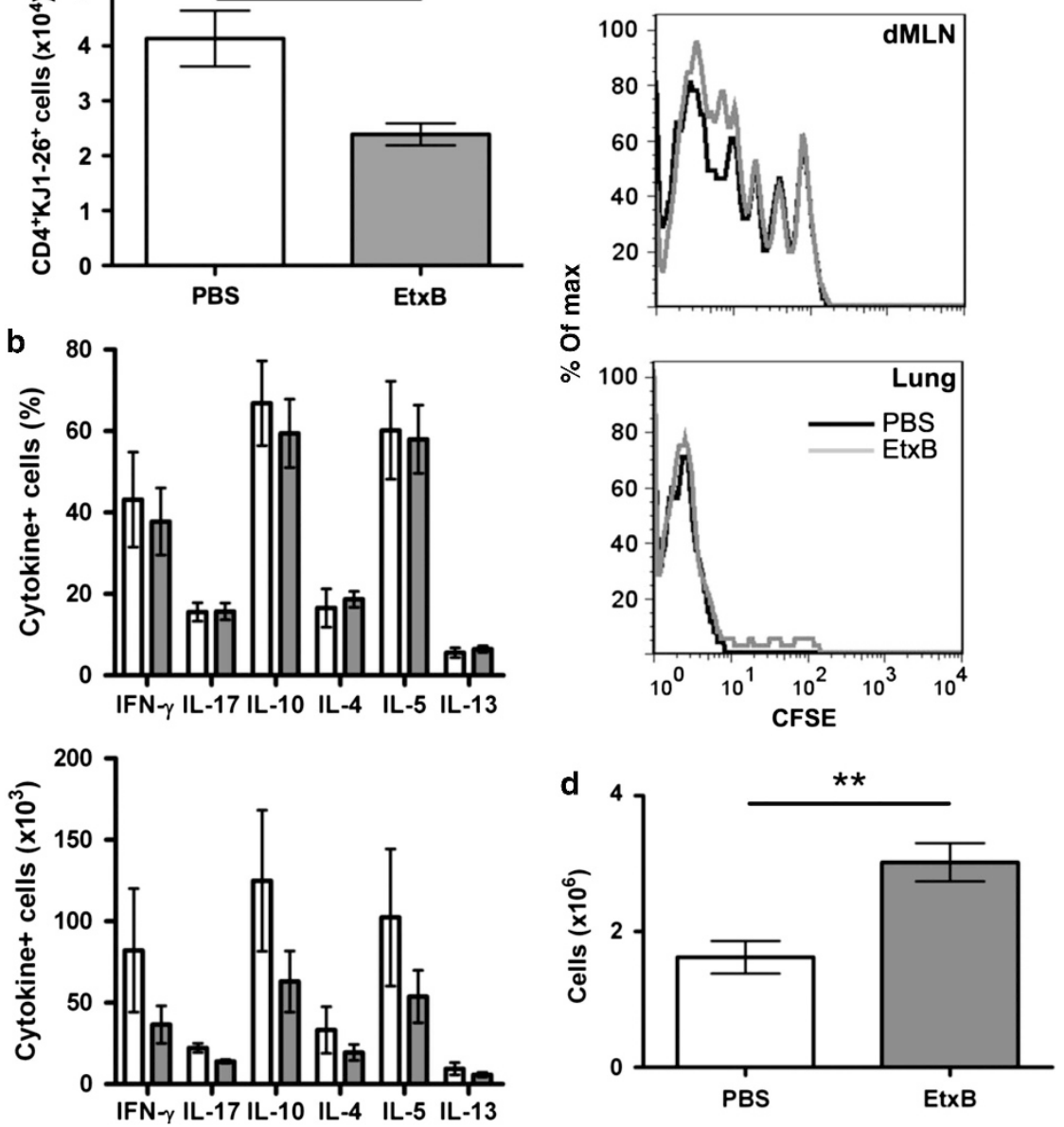

Figure 5 The effect of EtxB (B subunit of E. coli heat-labile enterotoxin) treatment on ovalbumin (OVA)-specific CD4 ${ }^{+}$T-cell lung infiltration, cytokine secretion, and proliferation. In all, $5 \times 10^{6}$ OVA peptide-specific CD4 ${ }^{+}$T cells were adoptively transferred into OVA-sensitized mice on the day of the second sensitization. Mice were then treated with $50 \mu \mathrm{g} \mathrm{EtxB}$ or an equivalent volume of phosphate-buffered saline (PBS) on 4 consecutive days, before aerosol challenge with OVA for 6 consecutive days. Following challenge, cells were isolated from the lung and (a) the total OVA-specific $\mathrm{CD}^{+}$T-cell numbers were determined $(n=5)$. Cells were also restimulated in vitro with phorbol myristate acetate and ionomycin and (b) the relative proportion and total number of OVA peptide-specific CD4 ${ }^{+}$T cells secreting T-cell-associated cytokines determined by flow cytometry $(n=4)$. In a separate experiment, OVA-specific CD4 ${ }^{+}$T cells were labeled with CFSE (carboxyfluorescein succinimidyl ester) before adoptive transfer and (c) proliferation of the OVA-specific CD4 ${ }^{+}$T-cell population of the draining mediastinal lymph nodes (dMLN) and lung determined by flow cytometry (histogram shown is representative of five mice). (d) The effect of EtxB on lung infiltration was also assessed by removing and counting isolated lung mononuclear cells following EtxB or PBS treatment in the absence of OVA aerosol challenge $(n=5)$. The data shown are representative of two experiments, bars represent mean \pm s.e.m.. Statistical differences were determined using Student's -test, ${ }^{\star} P<0.05,{ }^{\star \star} P<0.01$. IFN, interferon; IL, interleukin.

pool was a part of the process of immune modulation. To do this, a population of naive OVA-specific $\mathrm{CD} 4^{+} \mathrm{T}$ cells were adoptively transferred into OVA-sensitized mice on the day of the second intraperitoneal sensitization. Recipients were then given intranasal EtxB or PBS on 4 consecutive days. On day 6 following treatment, cells from the CLN and spleens of these mice were stained with antibodies against CD4, Foxp3, and the OVA-specific TCR for flow cytometric analyses. In addition, in order to investigate whether numbers of OVA-specific Treg are increased in the affected tissue, similar analysis was carried out on cells isolated from the lung and the dMLN. Intranasal treatment with EtxB clearly induced a significant increase in the proportion of OVA-specific CD4 ${ }^{+} \mathrm{T}$ cells that express Foxp3 in both the CLN and the spleen (Figure 8a). Similarly, in both the lung and the dMLN, an increased proportion of OVA-specific Foxp3-expressing $\mathrm{CD}^{+}{ }^{+} \mathrm{T}$ cells was observed following EtxB treatment (Figure 8b). Therefore, EtxB modulates differentiation of the antigen-specific T-cell pool, inducing increased Treg cell numbers, which are found both systemically and at the local site of inflammation.

\section{DISCUSSION}

In this study, intranasal EtxB treatment was shown to be capable of preventing lung inflammation in mice. As we previously reported in arthritis, disease prevention correlated with an increased proportion of Foxp3-expressing CD $4{ }^{+}$Treg. In the present study, data indicate that this pool of Treg include cells that are specific for the antigen driving the pathological 
response, which alter their differentiation pathway to become Foxp $3^{+}$as a result of treatment. Our findings implicate this as the common mechanism underlying the ability of EtxB to
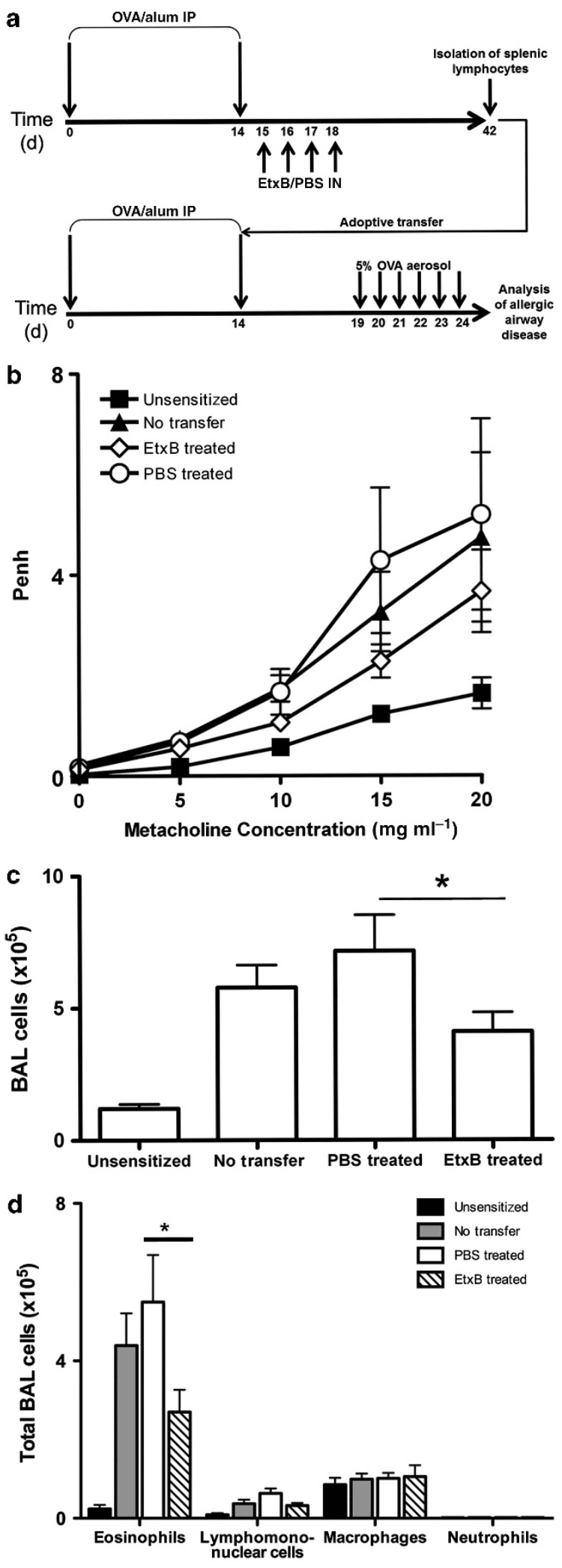

prevent both autoimmune and allergic diseases despite their obvious differences in target tissue and Th cell profile. There is now increasing evidence suggesting that human autoimmune disease and allergy is associated with a deficiency in the function of Treg, ${ }^{21-23}$ and thus the fact that EtxB modulates Treg implies that it potentially could be used to treat a range of human immune-mediated pathologies.

The role of Treg in the modulating allergy has now been clearly established. Reduced numbers and functionality of Treg has been observed in asthmatic patients ${ }^{24}$ and administration of allergens to patients has been associated with the generation of $\mathrm{CD}^{+}{ }^{+} \mathrm{T}$ cells with regulatory qualities. ${ }^{25,26}$ Further evidence for Treg involvement comes from animal studies in which depletion of $\mathrm{CD} 4{ }^{+} \mathrm{CD} 25^{+}$T cells from mice strains normally resistant to asthma induction permits the development of asthmatic symptoms ${ }^{27}$ and exacerbates these in asthmasensitive strains. ${ }^{28}$ Additionally, adoptive transfer of OVAspecific $\mathrm{CD}^{+}{ }^{+} \mathrm{CD} 25^{+}$Treg can prophylactically inhibit the development of asthma through a mechanism dependent on IL- $10^{29}$ as well as inhibit the further development of symptoms of chronic asthma, such as airway remodeling. ${ }^{30}$ The ability to EtxB to enhance Treg cell numbers, and increase the proportion of allergen-specific T cells with a Treg phenotype is therefore consistent with its capacity to inhibit pathological changes in the lung. As we have shown in both EtxB-mediated protection from CIA and T1D, ${ }^{13,14}$ adoptive transfer of $\mathrm{T}$ cells from treated animals recapitulated disease protection, further linking the observed Treg induction to the process of disease protection. Although adoptive transfer of $\mathrm{CD} 4{ }^{+} \mathrm{T}$ cells from EtxB-treated mice was not as effective at suppressing lung changes as treatment with EtxB, this may have been due to the small number of OVA-specific CD4 ${ }^{+}$Treg that are likely to be in a population of polyclonal splenic $\mathrm{CD} 4^{+} \mathrm{T}$ cells. However, a direct effect of EtxB in modulating the lung microenvironment following intranasal treatment may have also contributed to EtxB-mediated protection.

Direct treatment of bone marrow-derived or freshly isolated mononuclear phagocyte populations with EtxB does not drive Foxp3 expression in CD $4^{+} \mathrm{T}$ cells (data not shown). We believe

Figure 6 The effect of adoptive transfer of $C D 4^{+} T$ cells from EtxB $(B$ subunit of $E$. coli heat-labile enterotoxin)-treated mice on the development of asthma. (a) Ovalbumin (OVA)-sensitized mice were intranasally (IN) treated with $50 \mu \mathrm{g} \mathrm{EtxB}$ or an equivalent volume of phosphate-buffered saline (PBS) on 4 consecutive days. On day 24 post-treatment, splenic $\mathrm{CD} 4^{+} \mathrm{T}$ cells were purified by MACS and adoptively transferred into OVA-sensitized recipients on the day of the second sensitization. Groups of mice that were unsensitized or sensitized and received no transfer were included as controls. Four days after transfer, all groups were challenged with 5\% OVA aerosol for 6 consecutive days.

(b) Following challenge, enhanced pause (Penh) values were determined by recording respiratory pressure curves by unrestrained whole-body plethysmography in response to increasing concentrations of methacholine. (c) Bronchoalveolar lavage (BAL) cells were also isolated, counted, stained with Leishmans stain, and (d) the numbers of eosinophils, lymphomononuclear cells, macrophages, and neutrophils were determined by microscopy. Values/bars represent mean \pm s.e.m. $(n=8)$. Statistical differences were determined using analysis of variance with a Tukey post-hoc test $\left({ }^{\star} P<0.05\right)$. IP, intraperitoneal. 

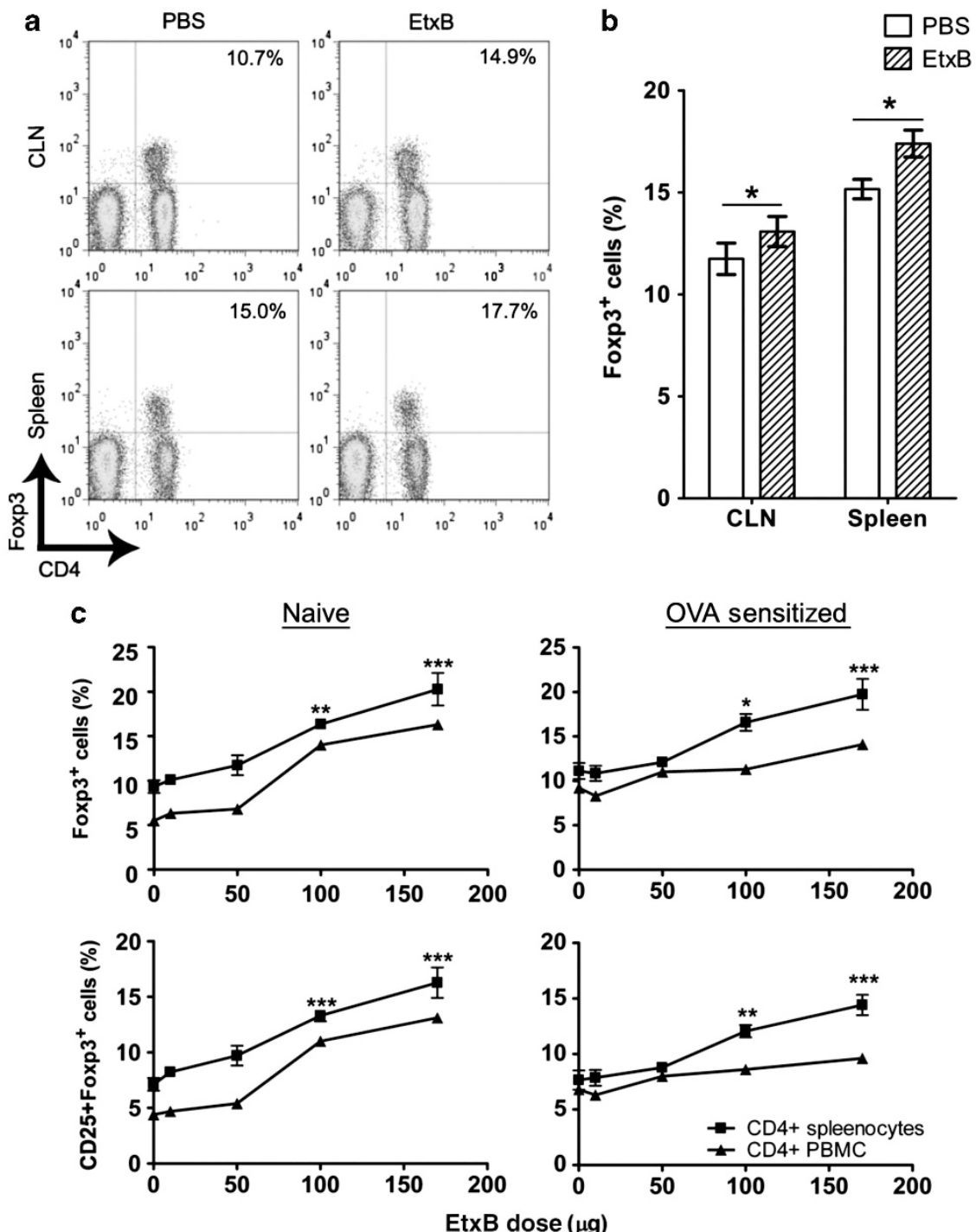

Figure 7 Effect of intranasal EtxB (B subunit of E. coli heat-labile enterotoxin) treatment on Foxp3 expression. Mice were intranasally treated with $20 \mu \mathrm{g}$ of EtxB or an equivalent volume of phosphate-buffered saline (PBS) on 4 consecutive days. (a) On day 6 post-treatment, single-cell suspensions were prepared from the cervical lymph nodes (CLN) and spleen, which were stained for CD4 and Foxp3 and analyzed by flow cytometry. Representative data are shown. (b) The percentage of Foxp3-expressing cells within the CD4 ${ }^{+}$T-cell population was determined $(n=6)$. In order to determine whether this effect was dose dependent, naive and ovalbumin (OVA)-sensitized mice were intranasally treated with a variety of EtxB doses $(10,50,100$, and $170 \mu \mathrm{g})$ or an equivalent volume of PBS on 4 consecutive days. On day 5 post-treatment, single-cell suspensions were prepared from the spleen and peripheral blood mononuclear cells (PBMC) isolated. These were stained for CD4, CD25 and Foxp3 and analyzed by flow cytometry. (c) The percentage of Foxp $3^{+}$and $\mathrm{CD}_{2} 5^{+} \mathrm{Foxp}^{+}$cells in the CD4 ${ }^{+}$population was determined $(n=4$ for the spleen, PBMC value represents four pooled mice). The data shown are representative of at least three similar experiments, bars/values represent mean \pm s.e.m.. Statistical differences was determined using Student's $t$-test (b) or analysis of variance with a Tukey post-hoc test (c) $\left({ }^{*} P<0.05,{ }^{* *} P<0.01,{ }^{* * *} P<0.001\right)$.

that this reflects the fact that multiple activities of EtxB that occur in vivo are involved in EtxB-mediated Treg induction. Importantly, our previous studies have indicated that Treg induction following EtxB treatment is dependent on both IL-10 and TGF- $\beta$, both of which are induced in epithelial cells at the site of delivery as well as by mononuclear cells in associated lymphoid tissues. ${ }^{18}$ We believe that these effects, possibly together with other activities, modulate local tissue microenvironments creating conditions that favor the differentiation of recently activated T cells into a pathway that generates Treg.
As with any activated effector cell, Treg induced in this way would migrate to the sites of inflammation where they may exert their regulatory influence in the local environment. The data presented here are consistent with this hypothesis. CFSE studies highlighted that only $\mathrm{T}$ cells that have undergone multiple cell divisions enter the lung. EtxB treatment led to an increase in the proportion of OVA-specific $\mathrm{CD}^{+}{ }^{+} \mathrm{T}$ cells expressing Foxp 3 in local and systemic lymphoid tissues and an increased proportion of these cells were observed in the lung. Treatment was associated with a decrease in immune cell 

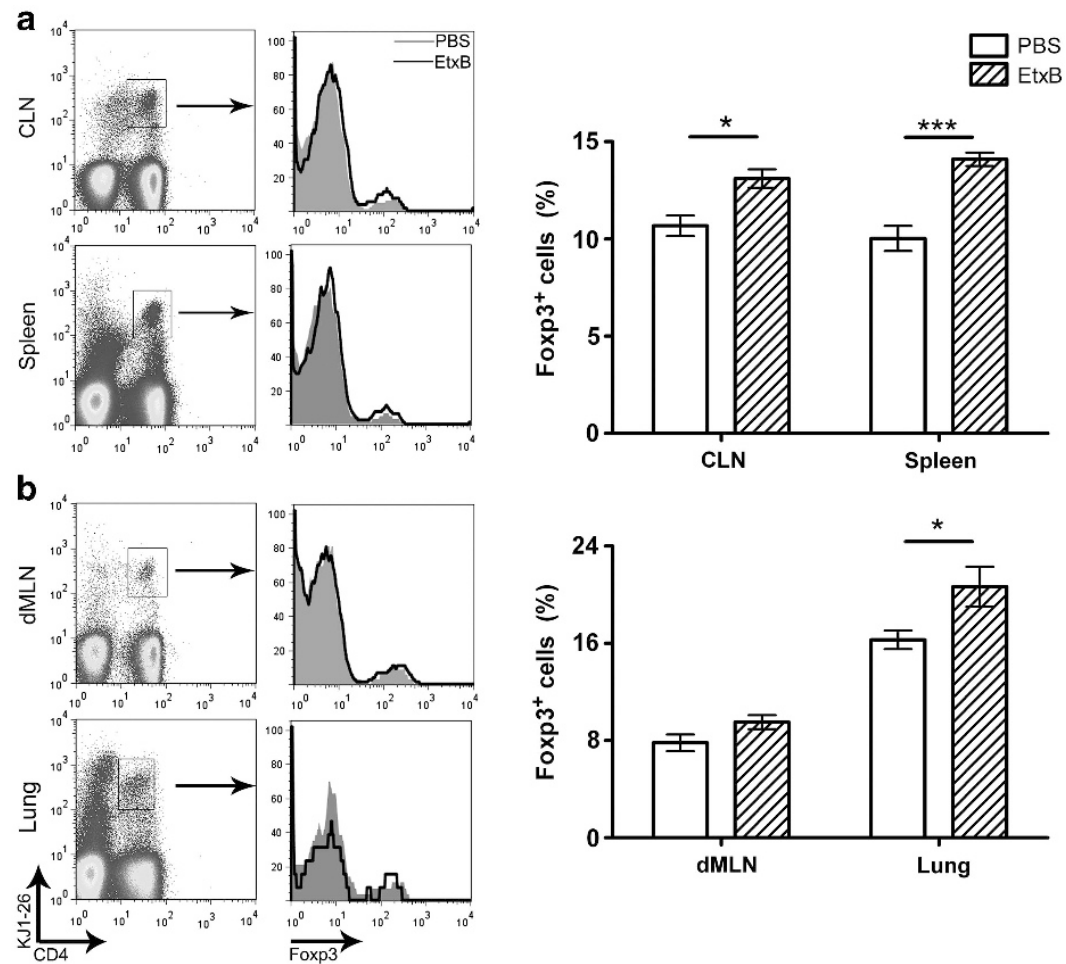

Figure 8 Effect of intranasal EtxB (B subunit of E. coli heat-labile enterotoxin) treatment on Foxp3 expression within ovalbumin (OVA)-specific CD4 ${ }^{+}$T-cell populations. In all, $5 \times 10^{6}$ OVA-specific T cells from DO11.10 mice were adoptively transferred into OVA-sensitized mice immediately before the second sensitization. Following sensitization, mice were intranasally treated with $50 \mu \mathrm{g}$ of EtxB or an equivalent volume of phosphate-buffered saline (PBS) on 4 consecutive days. On day 5 post-treatment, single-cell suspensions were prepared from the (a) cervical lymph nodes (CLN) and spleen or (b) draining mediastinal lymph nodes (dMLN) and lung. Foxp3 ${ }^{+}$cells within the OVA-specific CD4 ${ }^{+}$ $\mathrm{T}$ cell population were determined by flow cytometry. The data shown are representative of two similar experiments, bars represent mean \pm s.e.m. $(n=5)$. Statistical differences were determined using Student's $t$-test $\left({ }^{\star} P<0.05,{ }^{* * \star} P<0.001\right)$.

infiltration of the lung as determined in samples of BAL and in lung homogenates, and reduced cytokine production by those OVA-specific T cells that were present. In addition, there was a clear decrease in the concentration of IL-4 in the BAL.

Although our observations of responses in the BAL and lung are consistent with a mechanism of action involving Treg entering the local tissue and exerting suppressive effects on the pathological response, other processes may contribute to the ability of EtxB to mediate disease protection. Such effects may include systemic modulation of the anti-OVA response and a direct effect of EtxB on the local lung tissue microenvironment. Evidence for systemic modulation of the anti-OVA immune response came from the suppression of IL-4 production by spleen cells re-stimulated in vitro with OVA following treatment of OVA-sensitized animals. Similarly, our data from CIA demonstrated suppressed IFN- $\gamma$ production following re-stimulation of splenocytes from collagen-sensitized EtxB-treated mice. ${ }^{13}$ The systemic effects are also consistent with the activity of Treg cells induced by EtxB. We have shown that the proportion of Foxp $3^{+} \mathrm{T}$ cells is increased in the spleen, blood, CLN, and dMLN. Their presence is therefore likely to affect the differentiation of $\mathrm{T}$ cells being activated in response to antigen sensitization, which would still be on-going following the first sensitization with OVA/alum, and is induced by the second sensitization with OVA/alum, the timing of which coincided with the commencement of treatment.

Whether or not the protection observed in these studies was mediated in part by a direct effect of EtxB on the lung is unclear. Protection from CIA and T1D followed intranasal, intragastric, or subcutaneous treatment with EtxB. The extremely high binding affinity of EtxB for its receptors precludes systemic exposure to free protein, which is consistent with our failure to detect EtxB in serum, ${ }^{18}$ and our observation that it is found abundantly in mucosal tissue local to the site of delivery as well as in/on immune cells locally and systemically. Thus, a direct effect on the inflamed tissue was clearly not critical in prevention of autoimmune disease. However, in the current studies, treatment was given intranasally, and it is likely that a proportion of the administered EtxB reaches the lung. Adoptive transfer was not fully able to mimic the effects of EtxB treatment, and intragastric treatment with EtxB was only partially able to prevent asthma (data not shown).

EtxB is itself immunogenic, ${ }^{31}$ and other studies have suggested that intrapulmonary delivery of an immunogenic whole-toxin mutant, LTK63, can modulate the cellular environment in the lung, ${ }^{32}$ leading to protection from pulmonary viral and fungal infection. ${ }^{33}$ Furthermore, EtxB causes local production of IL-10 and TGF- $\beta_{1}$ by epithelial and

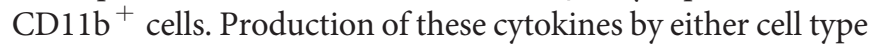


in the lung could have a role in modifying the environment to suppress lung pathology. ${ }^{34,35}$ An increased concentration of IL-10 in the BAL was linked to the suppression of asthma following adoptive transfer of OVA-specific $\mathrm{CD} 4{ }^{+} \mathrm{CD} 25^{+}$ Treg, ${ }^{29}$ and our studies showed a slight increase in IL-10 in the BAL of treated animals. IL-10 can suppress the secretion of other cytokines and control cellular activation, consistent with modulating pathology. In addition, IL-10 permits the Foxp3inducing capacity of TGF- $\beta_{1}{ }^{36}$ and hence could further elevate Treg differentiation. Although TGF- $\beta_{1}$ is considered as an immune suppressive cytokine, and as a result local production may help modulate asthma, its role is clearly more complex. The combination of IL- 4 and TGF- $\beta_{1}$ has been shown to induce IL-9-secreting $\mathrm{CD}^{+} \mathrm{T}$ cells, ${ }^{37,38}$ a cytokine known to exacerbate asthmatic symptoms. ${ }^{39}$ Further, TGF- $\beta_{1}$ has a clear role in causing tissue remodeling changes that are associated with decreased airway responsiveness in chronic asthma. ${ }^{40}$

Taken together, the results of this study demonstrate that EtxB treatment is capable of suppressing a Th2-mediated pathology in addition to its reported ability to control Th1mediated autoimmune diseases. This establishes it as a potential therapeutic for a wide range of inflammatory diseases. The finding that the predominant mechanism by which EtxB mediates disease modification is through the induction of disease antigen-specific Treg is of particular significance. A number of studies have now clearly linked Treg defects as predisposing factors in many inflammatory diseases, raising interest in therapies that could redress the balance. To date, most approaches rely on a clear understanding of the precise antigens that are underlie disease such that they, or peptides derived from them can be used as part of the therapy. The fact that EtxB achieves this effect in the absence of antigen co-administration is of particular significance for its therapeutic potential. A bias towards the induction of Treg responses specific for antigens that are inducing pathogenic $\mathrm{CD} 4{ }^{+} \mathrm{T}$-cell responses at the time of treatment means that EtxB may be able to not only treat immune pathologies of complex or unknown etiologies, such as asthma, it also avoids the generation of Treg responses specific for antigens to which tolerance is undesirable.

\section{METHODS}

Experimental animals. Female BALB/c and DO11.10 mice (bred at University of Bristol animal facilities) were housed under barriermaintained conditions. Animals were cared for in accordance with the Animals (Scientific Procedure) Act 1986 of the United Kingdom. In order to sensitize mice, OVA (Sigma, Poole, UK) precipitated in alum (Sigma) was injected intraperitoneally on days 0 and 14. EtxB (or PBS as vehicle control) was administered in a $50 \mu \mathrm{l}$ volume by intranasal instillation as previously described ${ }^{41}$ on 4 consecutive days (days 1518). Preliminary studies showed that doses from $10-50 \mu \mathrm{g}$ were effective in modulating responses and therefore the dose delivered was within this range, and is stated. Following treatment, mice were challenged with a 5\% OVA (in PBS) aerosol for 6 consecutive days (days 19-24). All readouts, unless otherwise stated, were performed on day 25. Adoptive transfer of nylon wool-enriched splenic DO11.10 $\mathrm{CD}^{+} \mathrm{T}$ cells (routinely $>65 \% \mathrm{CD}^{+}$) and MACS-purified (negatively selected, performed as per the manufacturer's (Miltinyi Biotech, Bisley, UK) instructions) $\mathrm{CD}^{+}{ }^{+} \mathrm{T}$ cells (routinely $>90 \%$
$\mathrm{CD}^{+}$) was achieved by intravenous tail vein injection before the second sensitization (day 14).

Unrestrained whole-body plethysmography. The Penh was determined by recording respiratory pressure curves by unrestrained whole-body plethysmography (Buxco Research Systems, Winchester, $\mathrm{UK}$ ) in response to increasing concentrations of methacholine (Sigma) for 3 min each. ${ }^{42}$

BAL collection and analysis. Mice were killed by terminal anesthesia and three washes of $400 \mu \mathrm{l}$ PBS were introduced into the lung through the trachea and pooled. BAL samples were centrifuged and the supernatants reserved for further analysis. The remaining cells were counted and cytospins were prepared. Cytospins were stained with Leishmans stain (VWR, Lutterworth, UK), followed by washing in Sorensons Buffer (PBC, containing 4\% paraformaldehyde). Five areas were analyzed visually for the presence of macrophages, neutrophils, lymphomononuclear cells, and eosinophils. The mean proportion of each cell type was determined and multiplied by the cell number to determine the total cell number for each cell type.

Measurement of antibody titers and cytokine concentrations. Antibody titers and cytokine concentrations in BAL supernatants and serum were determined by ELISA as previously described. ${ }^{13,43}$ For antibody titers, plates were coated with OVA, before blocking with $1 \%$ bovine serum albumin in PBS (1\% BSA-PBS; Sigma). Serial dilutions of samples were added and incubated for $2 \mathrm{~h}$ at $37^{\circ} \mathrm{C}$. Bound OVAspecific Ig was detected using horseradish peroxidase-conjugated antimouse $\operatorname{IgG}_{1}, \operatorname{IgG}_{2 \mathrm{a}}$ or IgE (all AbD Serotec, Kidlington, UK). Plates were developed with $o$-phenylenediamine dihydrochloride substrate (Sigma), and the optical density was measured $(490 \mathrm{~nm})$. Endpoint titers were determined by linear regression analysis of $\log _{10}$-transformed data using Statistics (version W1.58) (Blackwell Scientific Publications, Oxford, UK).

For the determination of cytokine concentrations by ELISA, plates were coated with purified anti-mouse IFN- $\gamma$, IL-4, or IL-10 (Invitrogen, Paisley, UK), blocked with 1\% BSA-PBS, and detected using biotinylated anti-mouse IFN- $\gamma$, IL-4, or IL-10 (Invitrogen) and a streptavidin-horseradish peroxidase conjugate (Sigma). Plates were developed with tetramethylbenzidine (Insight Biotechnology, Wembley, UK) and the absorbance at $450 \mathrm{~nm}$ was determined. Luminex analysis of IFN- $\gamma$, IL-4, IL-10, and IL-13 was performed according to the manufacturer's instructions (Millipore, Watford, UK). Standards and samples were run in duplicate and at least 50 microspheres were analyzed per sample. Cytokine concentrations were calculated using standard curves of recombinant mouse cytokines.

Cell preparation and restimulation. Single-cell suspensions were prepared from the CLN, dMLN, and spleen by mechanical disruption. Red blood cells were removed by ammonium chloride lysis. Enzymatic digestion of the lung was performed by incubating minced tissue in Liberase blendzyme $3\left(0.14 \mathrm{U} \mathrm{ml}^{-1}\right.$; Roche, Burgess Hill, UK) and DNase $1\left(0.1 \mathrm{mg} \mathrm{ml}^{-1}\right.$; Sigma) for $45 \mathrm{~min}$ at $37^{\circ} \mathrm{C}$ before mechanical disruption. ${ }^{44}$ Samples were overlaid with an equal volume of Histopaque 1083 (Sigma) before centrifugation and isolation.

In order to determine cytokine secretion and proliferation, cells were incubated in the presence or absence of OVA peptide (323-339, ISQAVHAAHAEINEAGR; University of Bristol) for 4 days, after which supernatants were removed. Proliferation was determined by $3 \mathrm{H}$-thymidine incorporation. For intracellular cytokine staining, cells were incubated in RPMI 1640 (Invitrogen) containing 10\% fetal calf serum (Invitrogen), $100 \mathrm{U} \mathrm{ml}^{-1}$ penicillin $/ 100 \mu \mathrm{g} \mathrm{ml}^{-1}$ streptomycin (Sigma), $20 \mathrm{~mm}$ L-glutamine (Sigma), $100 \mathrm{ng} \mathrm{ml}^{-1}$ phorbol myristate acetate (Sigma), $500 \mathrm{~mm}$ ionomycin (Sigma) and $1 \mu \mathrm{g} \mathrm{ml}^{-1}$ Gogliplug (BD Biosciences, Oxford, UK) for $4 \mathrm{~h}$ before staining.

Flow cytometry. Antibodies used for flow cytometric analyses included anti-mouse CD16/CD32 (2.4G2), fluorescein isothiocyanate, and phycoerythrin-conjugated anti-mouse CD4 (H129.19), 
phycoerythrin-conjugated anti-mouse CD25 (PC61), anti-mouse IFN- $\gamma$ (XMG1.2), anti-mouse IL-10 (JES5-16E3) (all BD Biosciences), and anti-mouse IL-5 (TRFK5), Alexa Flour 647 conjugated anti-mouse IL-13 (eBio13A) and anti-mouse IL-4 (11B11), antigen-presenting cell-conjugated anti-mouse IL-17A (eBio17B7), and anti-mouse Foxp3 (FJK-16s; all eBioscience, San Diego, CA), and Tricolorconjugated anti-DO11.10 TCR (KJ1-26; Invitrogen).

Before staining, Fc receptors were blocked with anti-CD16/32 antibody. For surface staining, cells were incubated with appropriate combinations of antibodies for $30 \mathrm{~min}$ at $4{ }^{\circ} \mathrm{C}$. Intracellular cytokine and Foxp3 staining was performed using the Fixation and Permeabilization buffers as per the manufacturer's instructions (eBioscience). Stained cells were analyzed on a FACSCalibur flow cytometer (BD biosciences). At least $1 \times 10^{5}$ events and $1 \times 10^{6}$ events were collected for each sample of Foxp3 staining in BALB/c mice and detection of DO11.10 CD4 ${ }^{+} \mathrm{T}$ cells, respectively. Collected data files were analyzed with Flowjo (Treestar, Ashland, OR).

Statistical analyses. Results are expressed as the mean \pm s.e.m. unless otherwise indicated. Statistical analyses (either Student's $t$-test or ANOVA with a Tukey post-hoc test where indicated) were performed using Graphpad Prism 4 (Graphpad Software, San Diego, CA).

\section{ACKNOWLEDGEMENTS}

This work was supported by grants from the Medical Research Council and Trident Pharmaceuticals Inc. We thank Professor Clare Lloyd (Imperial College, London) for help in the establishment of the asthma model, and Rachel Williams for production of the EtxB.

\section{DISCLOSURE}

Dr Williams is a shareholder in Trident Pharmaceuticals, which holds patent rights to the use of EtxB as a treatment for inflammatory disease. The other authors declare no conflicts of interest.

(c) 2013 Society for Mucosal Immunology

\section{REFERENCES}

1. Eder, W., Ege, M.J. \& von Mutius, E. The asthma epidemic. N. Engl. J. Med. 355, 2226-2235 (2006).

2. Hamid, Q. \& Tulic, M. Immunobiology of asthma. Annu. Rev. Physiol. 71, 489-507 (2009).

3. Fanta, C.H. Asthma. N. Engl. J. Med. 360, 1002-1014 (2009).

4. Sakaguchi, S., Sakaguchi, N., Asano, M., Itoh, M. \& Toda, M. Immunologic self-tolerance maintained by activated $T$ cells expressing IL-2 receptor alpha-chains (CD25). Breakdown of a single mechanism of self-tolerance causes various autoimmune diseases. J. Immunol. 155, 1151-1164 (1995).

5. Xystrakis, E. et al. Reversing the defective induction of IL-10-secreting regulatory Tcells in glucocorticoid-resistant asthma patients. J. Clin. Invest. 116, 146-155 (2006).

6. Bauer, L. et al. Modulation of the allergic immune response in BALB/c mice by subcutaneous injection of high doses of the dominant Tcell epitope from the major birch pollen allergen Bet v 1. Clin. Exp. Immunol. 107, 536-541 (1997).

7. von Garnier, C. et al. Allergen-derived long peptide immunotherapy downregulates specific IgE response and protects from anaphylaxis. Eur. J. Immunol. 30, 1638-1645 (2000).

8. Alexander, C., Ying, S., B Kay, A. \& Larche, M. Fel d 1-derived Tcell peptide therapy induces recruitment of CD4 + CD25 +; CD4 + interferongamma $+T$ helper type 1 cells to sites of allergen-induced late-phase skin reactions in cat-allergic subjects. Clin. Exp. Allergy 35, 52-58 (2005).

9. Verhoef, A., Alexander, C., Kay, A.B. \& Larche, M. T cell epitope immunotherapy induces a CD4 + Tcell population with regulatory activity. PLoS Med. 2, e78 (2005)

10. Campbell, J.D. et al. Peptide immunotherapy in allergic asthma generates IL-10-dependent immunological tolerance associated with linked epitope suppression. J. Exp. Med. 206, 1535-1547 (2009).

11. Larche, M. Update on the current status of peptide immunotherapy. J. Allergy Clin. Immunol. 119, 906-909 (2007).
12. Ober, C. \& Thompson, E.E. Rethinking genetic models of asthma: the role of environmental modifiers. Curr. Opin. Immunol. 17, 670-678 (2005).

13. Luross, J.A., Heaton, T., Hirst, T.R., Day, M.J. \& Williams, N.A. Escherichia coli heat-labile enterotoxin B subunit prevents autoimmune arthritis through induction of regulatory CD4 $+\mathrm{T}$ cells. Arthritis Rheum. 46, 1671-1682 (2002)

14. Ola, T.O. \& Williams, N.A. Protection of non-obese diabetic mice from autoimmune diabetes by Escherichia coli heat-labile enterotoxin B subunit. Immunology 117, 262-270 (2006).

15. Spangler, B.D. Structure and function of cholera toxin and the related Escherichia coli heat-labile enterotoxin. Microbiol. Rev. 56, 622-647 (1992).

16. Sixma, T.K. et al. Refined structure of Escherichia coli heat-labile enterotoxin, a close relative of cholera toxin. J. Mol. Biol. 230, 890918 (1993).

17. Cassel, D. \& Pfeuffer, T. Mechanism of cholera toxin action: covalent modification of the guanyl nucleotide-binding protein of the adenylate cyclase system. Proc. Natl. Acad. Sci. USA 75, 2669-2673 (1978).

18. Donaldson, D.S., Tong, K.K. \& Williams, N.A. Mucosal administration of the $B$ subunit of $E$. coli heat-labile enterotoxin promotes the development of Foxp3-expressing regulatory Tcells. Mucosal Immunol. 4, 227-238 (2011).

19. Brusselle, G.G. et al. Attenuation of allergic airway inflammation in IL-4 deficient mice. Clin. Exp. Allergy 24, 73-80 (1994).

20. Korsgren, M., Erjefalt, J.S., Korsgren, O., Sundler, F. \& Persson, C.G. Allergic eosinophil-rich inflammation develops in lungs and airways of B cell-deficient mice. J. Exp. Med. 185, 885-892 (1997).

21. Ling, E.M. et al. Relation of CD4 + CD25 + regulatory T-cell suppression of allergen-driven T-cell activation to atopic status and expression of allergic disease. Lancet 363, 608-615 (2004).

22. Thunberg, S. et al. Immune regulation by CD4 $+\mathrm{CD} 25+\mathrm{T}$ cells and interleukin-10 in birch pollen-allergic patients and non-allergic controls. Clin. Exp. Allergy 37, 1127-1136 (2007).

23. Brusko, T.M., Putnam, A.L. \& Bluestone, J.A. Human regulatory Tcells: role in autoimmune disease and therapeutic opportunities. Immunol. Rev. 223, 371-390 (2008).

24. Hartl, D. et al. Quantitative and functional impairment of pulmonary $\mathrm{CD} 4+\mathrm{CD} 25$ hi regulatory $\mathrm{T}$ cells in pediatric asthma. J. Allergy Clin. Immunol. 119, 1258-1266 (2007).

25. Francis, J.N., Till, S.J. \& Durham, S.R. Induction of IL-10 + CD4 + CD25+ T cells by grass pollen immunotherapy. J. Allergy Clin. Immunol. 111, 1255-1261 (2003)

26. Gardner, L.M., Thien, F.C., Douglass, J.A., Rolland, J.M. \& O'Hehir, R.E. Induction of $\mathrm{T}$ 'regulatory' cells by standardized house dust mite immunotherapy: an increase in CD4 + CD25 + interleukin-10 + T cells expressing peripheral tissue trafficking markers. Clin. Exp. Allergy 34, 1209-1219 (2004)

27. Lewkowich, I.P. et al. CD4 + CD25 + Tcells protect against experimentally induced asthma and alter pulmonary dendritic cell phenotype and function. J. Exp. Med. 202, 1549-1561 (2005).

28. Leech, M.D., Benson, R.A., De Vries, A., Fitch, P.M. \& Howie, S.E. Resolution of Der p1-induced allergic airway inflammation is dependent on CD4 + CD25 + Foxp3 + regulatory cells. J. Immunol. 179, 7050-7058 (2007).

29. Kearley, J., Barker, J.E., Robinson, D.S. \& Lloyd, C.M. Resolution of airway inflammation and hyperreactivity after in vivo transfer of CD4 + CD25 + regulatory Tcells is interleukin 10 dependent. J. Exp. Med. 202, 1539-1547 (2005).

30. Kearley, J., Robinson, D.S. \& Lloyd, C.M. CD4 + CD25 + regulatory Tcells reverse established allergic airway inflammation and prevent airway remodeling. J. Allergy Clin. Immunol. 122, 617-624 e616 (2008).

31. Nashar, T.O., Webb, H.M., Eaglestone, S., Williams, N.A. \& Hirst, T.R. Potent immunogenicity of the B subunits of Escherichia coli heat-labile enterotoxin: receptor binding is essential and induces differential modulation of lymphocyte subsets. Proc. Natl. Acad. Sci. USA 93, 226-230 (1996).

32. Tritto, E. et al. The acquired immune response to the mucosal adjuvant LTK63 imprints the mouse lung with a protective signature. J. Immunol. 179, 5346-5357 (2007).

33. Williams, A.E. et al. Innate imprinting by the modified heat-labile toxin of Escherichia coli (LTK63) provides generic protection against lung infectious disease. J. Immunol. 173, 7435-7443 (2004). 


\section{ARTICLES}

34. Fu, C.L., Chuang, Y.H., Chau, L.Y. \& Chiang, B.L. Effects of adenovirusexpressing IL-10 in alleviating airway inflammation in asthma. J. Gene Med. 8, 1393-1399 (2006).

35. Hansen, G. et al. CD4(+) Thelper cells engineered to produce latent TGFbeta1 reverse allergen-induced airway hyperreactivity and inflammation. J. Clin. Invest. 105, 61-70 (2000).

36. Fantini, M.C., Becker, C., Monteleone, G., Pallone, F., Galle, P.R. \& Neurath, M.F. Cutting edge: TGF-beta induces a regulatory phenotype in CD4 + CD25- T cells through Foxp3 induction and down-regulation of Smad7. J. Immunol. 172, 5149-5153 (2004).

37. Dardalhon, V. et al. IL-4 inhibits TGF-beta-induced Foxp3 + T cells and, together with TGF-beta, generates IL-9 + IL-10 + Foxp3(-) effector Tcells. Nat. Immunol. 9, 1347-1355 (2008).

38. Veldhoen, M. et al. Transforming growth factor-beta 'reprograms' the differentiation of Thelper 2 cells and promotes an interleukin 9-producing subset. Nat. Immunol. 9, 1341-1346 (2008).
39. McLane, M.P. et al. Interleukin-9 promotes allergen-induced eosinophilic inflammation and airway hyperresponsiveness in transgenic mice. Am. J. Respir. Cell Mol. Biol. 19, 713-720 (1998).

40. Makinde, T., Murphy, R.F. \& Agrawal, D.K. The regulatory role of TGF-beta in airway remodeling in asthma. Immunol. Cell Biol. 85, 348-356 (2007).

41. Turcanu, V., Hirst, T.R. \& Williams, N.A. Modulation of human monocytes by Escherichia coli heat-labile enterotoxin B-subunit; altered cytokine production and its functional consequences. Immunology 106, 316-325 (2002).

42. Hamelmann, E. et al. Noninvasive measurement of airway responsiveness in allergic mice using barometric plethysmography. Am. J. Respir. Crit. Care Med. 156 (3 Part 1), 766-775 (1997).

43. Apostolaki, M. \& Williams, N.A. Nasal delivery of antigen with the B subunit of Escherichia coli heat-labile enterotoxin augments antigen-specific T-cell clonal expansion and differentiation. Infect. Immun. 72, 4072-4080 (2004).

44. Tager, A.M. et al. Leukotriene B4 receptor BLT1 mediates early effector T cell recruitment. Nat. Immunol. 4, 982-990 (2003). 